\title{
RENDEZVOUS SEARCH WITH REVEALED INFORMATION: APPLICATIONS TO THE LINE
}

\author{
STEVE ALPERN, ${ }^{*}$ London School of Economics
}

\begin{abstract}
The symmetric rendezvous problem on a network $Q$ asks how two players, forced to use the same mixed strategy, can minimize their expected meeting time, starting from a known initial distribution on the nodes of $Q$. This minimum is called the (symmetric) 'rendezvous value' of $Q$. Traditionally, the players are assumed to receive no information while playing the game. We consider the effect on rendezvous times of giving the players some information about past actions and chance moves, enabling each of them to apply Bayesian updates to improve his knowledge of the other's whereabouts. This technique can be used to give lower bounds on the rendezvous times of the original game (without any revealed information). We consider the case in which they are placed a known distance apart on the line graph $Q$ (known as 'symmetric rendezvous on the line'). Our approach is to concentrate on a general analysis of the effect of revelations, rather than compute the best bounds possible with our technique.
\end{abstract}

Keywords: Rendezvous; search; common interest game; team theory

2000 Mathematics Subject Classification: Primary 90B40

\section{Introduction}

In this paper we consider how the revelation of information can affect an important unsolved problem in rendezvous theory: the symmetric rendezvous problem on the line or line graph (whose nodes are the integers, $\mathbb{Z}$ ) posed in [1], dubbed $\Gamma$. (In fact, given the recent solution by Weber [14] of the symmetric rendezvous problem on the complete graph $K_{3}, \Gamma$ is arguably the main unsolved problem.) The problem $\Gamma$ begins with a chance move $\alpha$, called the initial configuration. This move consists of both the placement of two players (rendezvousers), I and II, at nodes of $\mathbb{Z}$ a known distance (taken as 2) apart and their orientation (facing them to the left or to the right, independently). The random orientation models the assumption that the players do not have a common notion of direction along the line. The players then choose paths $s_{i}$ and $s_{j}$ with respect to their forward directions, using independent randomization from a common mixed strategy $\mu$. This constraint is what makes the game player symmetric, modeling the assumption that the players cannot meet beforehand to agree a joint strategy. (If they can, this is the playerasymmetric, or distinguishable-player, version studied in [2].) Given these choices, they arrive at some common node at a first (meeting) time $T \equiv T\left(s_{i}, s_{j}, \alpha\right)$. Their common aim is to minimize (under $\mu$ ) the expected value of the meeting time $T$, with the expectation taken with respect to both $\mu$ and the given distribution of $\alpha$, the initial configuration. This minimum time is called the (symmetric) rendezvous value, $v \equiv v(\Gamma)$, of $\Gamma$ and the minimizing distribution, $\bar{\mu}$, is called the optimal mixed strategy. For such symmetric rendezvous problems, it is equally valid to take the viewpoint that $\mu$ is either chosen by a single optimizer, such as the author

Received 7 June 2006; revision received 15 January 2007.

* Postal address: Department of Mathematics, London School of Economics, London WC2A 2AE, UK.

Email address: s.alpern@1se.ac.uk 
of a rendezvous protocol which is routinely given to people such as hikers, or programmed into a control unit of parallel computers to tell the players when and where to exchange their information. Such problems were first studied by Anderson and Weber [7] for the complete graphs $K_{m}$, and a general formulation, including continuous time and more complex notions of orientation, was subsequently given by the author [1].

Symmetric rendezvous problems on graphs [5] are traditionally studied under the assumption that each player receives no information about the other's whereabouts or strategy during the course of the play. In this paper we vary the 'no revealed information' assumption in such a way that, after a known number of moves $n$, both players receive a signal which depends on their actions and on chance moves up to that time. Suppose that their possible actions ( $n$-move sequences of forwards or backwards) are $s_{1}, \ldots, s_{m}$ and the initial chance moves are $\alpha_{1}, \ldots, \alpha_{K}$. The state of the game at time $n$ (i.e. after $n$ moves) depends on the triple $\left(s_{i}, s_{j}, \alpha_{k}\right)$, where $s_{i}$ and $s_{j}$ are the respective sequences chosen by players I and II, and 'Nature' (chance) chooses $\alpha_{k}$. For some triples $T\left(s_{i}, s_{j}, \alpha_{k}\right) \leq n$, in which case the game is already over at time $n$. For the rest, which form the uncertainty set $U \equiv U_{n}$, the game continues. The signal received by the players consists of the information of which element $P$ of a given partition $\mathcal{P}$ of $U$ contains the state $\left(s_{i}, s_{j}, \alpha_{k}\right)$. If the original rendezvous problem (game) is called $\Gamma$, then we call this revealed information version (where the players know $n$ and $\mathcal{P}$ from the outset) $\Gamma(n, \mathcal{P})$. After learning $P$, and knowing $s_{i}$, player I (for example) can update his priors on $\alpha$ and player II's action, $s_{j}$, given the knowledge of the common mixed strategy $\mu$ that both have used to pick their actions. The players then play optimally in the subgame $\Gamma(n, P)$, which, depending on $P$, may or may not be a symmetric game. The trick is to choose the partition $\mathcal{P}$ so that the optimal strategy in each subgame $\Gamma(n, P)$ can be determined. We can then ensure that the rendezvous value, $v(\mathcal{P})$, of the revealed information game $\Gamma(n, \mathcal{P})$ can be calculated (either definitively or in terms of $v$ ), yielding the inequality

$$
v(\mathcal{P}) \leq v
$$

because the additional information in $\mathcal{P}$ cannot increase the rendezvous time (it can always be ignored). The same observation shows that coarser partitions $\mathcal{P}$ give higher values of $v(\mathcal{P})$ and, hence, stronger lower bounds on the original rendezvous value $v$. Such lower bounds on rendezvous values are usually difficult to come by.

We apply these notions to the symmetric rendezvous problem on the line. The literature on this problem will be discussed in Section 2, and the application of revealed information to it will be analyzed in Sections 3 to 6, constituting the bulk of the paper. The only versions of revealed information in this context seem to be the papers of Baston and Gal [9] on the problem on the line, where each player learns his partner's starting point if he ever reaches it (by observing a marker left there), and Anderson and Weber [7] (who mentioned the possibility of players leaving messages at locations they have visited, but did not allow this in the versions of the problem on $K_{m}$ that they analyzed).

A revealed information version of [7], published at about the same time, is the paper on 'repeated coordination games' by Crawford and Haller [10], who considered the complete graph $K_{m}$ ( $m$ locations) with the players' locations (actions) revealed after each move. For small $m$, there are simple solutions. For $m=2$, the best the players can do is randomize forever on the two locations, meeting in Bernoulli expected time $1 / \frac{1}{2}=2$. This is equivalent to Anderson and Weber's result [7] for the complete graph $K_{2}$ on two nodes, since in that case the other player's location is 'revealed' (by the two not meeting). If $m=3$ and their first locations are distinct, then the players should both go to the unique location unoccupied during the first 
period, where they will meet at time 2 ; the least expected meeting time is thus

$$
\frac{1}{3} \times 1+\frac{2}{3}(1+1)=\frac{5}{3} .
$$

For $m=5$, two strategies are salient. Assuming that their initial locations are distinct, the players can (i) coordinate on these two locations forever (essentially playing the $m=2$ game), meeting in expected time $\frac{1}{5} \times 1+\frac{4}{5}(1+2)=\frac{13}{5}$, or they can (ii) go randomly among the three unoccupied locations and, if these are distinct, coordinate on the fifth location, with lower expected meeting time

$$
\frac{1}{5} \times 1+\frac{4}{5}\left(1+\frac{1}{3} \times 1+\frac{2}{3} \times 2\right)=\frac{7}{3} .
$$

\section{The symmetric rendezvous problem on the line}

In this section we formalize the description of $\Gamma$, the player-symmetric rendezvous problem on the line, and describe some of the literature giving upper bounds on $v$, the rendezvous value of $\Gamma$.

Problem $\Gamma$ begins with a chance move in which the two players are placed a distance $D=2$ apart on the real line (or two nodes away on the line graph $\mathbb{Z}$ ), and randomly faced in directions that each calls 'forward' forever. From an observer's perspective, player I is placed on the left of player II and Nature chooses equiprobably among four initial configurations, $\alpha_{k}, k=1,2,3,4$, each of which gives a \pm 1 -valued direction $d_{1}(k)$ to player I and $d_{2}(k)$ to player II (see Table 1). This chance move models the assumption that the players have no common notion of direction along the line, and that the first move of each must therefore be in a random direction, independent of the direction of their partner's first move.

Since we will need to display three-dimensional $(i, j, k)$ arrays, we will often include the final coordinate, $k$, in the $(i, j)$-labeled entries of a $2 \times 2$ array of the following form:

$$
\begin{array}{|l|l|l|}
\hline \alpha_{1}: \leftarrow \rightarrow & \leftarrow \alpha_{2}: \rightarrow \rightarrow \\
\hline \alpha_{3}: \leftarrow \leftarrow & \leftarrow \alpha_{4}: \rightarrow \leftarrow \\
\hline
\end{array}
$$

In each period $t=1,2, \ldots$, a player moves a distance of one unit in either his forward $(F)$ or backward $(B)$ direction. (A justification of this reduction to discrete moves can be found in [11] and, in a related context, [12].) A pure strategy is thus an infinite sequence of $F \mathrm{~s}$ and $B \mathrm{~s}$. (By symmetry of the line, we may assume without loss of generality that strategies begin with an $F$.) For numerical calculations, we take $F=+1$ and $B=-1$. If a player adopts strategy $s=(s(1), s(2), \ldots)$, with $s(t) \in\{F, B\}=\{+1,-1\}$, his net motion at time $t$ from his start, in his forward direction, is given by

$$
\hat{s}(t)=\sum_{r=1}^{t} s(r) .
$$

\begin{tabular}{|c|c|c|c|c|}
\hline$k$ & \multicolumn{2}{|c|}{$\alpha_{k}$} & $d_{1}$ & $d_{2}$ \\
\hline 1 & $\leftarrow$ & $\rightarrow$ & -1 & +1 \\
\hline 2 & $\rightarrow$ & $\rightarrow$ & +1 & +1 \\
\hline 3 & $\leftarrow$ & $\leftarrow$ & -1 & -1 \\
\hline 4 & $\rightarrow$ & $\leftarrow$ & +1 & -1 \\
\hline
\end{tabular}

TABLE 1: Initial configurations. 
If player I adopts strategy $s$, player II adopts strategy $s^{\prime}$, and Nature picks the initial configuration $\alpha_{k}$, then the directed distance from player II to player I at time $t$ (i.e. player II's location minus player I's location) is given by

$$
D\left(s, s^{\prime}, \alpha_{k}\right)(t)=\left(d_{2}(k) \hat{s}^{\prime}(t)+2\right)-\left(d_{1}(k) \hat{s}(t)\right),
$$

and the players therefore meet at time

$$
T\left(s, s^{\prime}, \alpha_{k}\right)=\min _{t}\left\{t: D\left(s, s^{\prime}, \alpha_{k}\right)(t)=0\right\} .
$$

Since $\alpha_{k}$ is picked equiprobably by Nature, their expected meeting time is given by the averaged function

$$
T\left(s, s^{\prime}\right)=\frac{1}{4} \sum_{k=1}^{4} T\left(s, s^{\prime}, \alpha_{k}\right)
$$

(which we give the same name, $T$ ). For example, if $s$ begins with $F F B$ and $s^{\prime}$ begins with $F B F$, it is easy to see that the meeting times corresponding to the four initial configurations $\alpha_{k}$, given in the two-dimensional ordering of (2), are

\begin{tabular}{|l|l|}
\hline$?$ & 2 \\
\hline$?$ & 1 \\
\hline
\end{tabular}

where the entry '?' signifies that the players have not met by time 3 ; that is, $T\left(s, s^{\prime}, k\right)>3$ for $k=1,3$.

The problem for the players (or for the author of a book which they each read for instructions) is to find the strategy which, when simultaneously adopted by both players, minimizes their expected meeting time. Clearly no pure strategy $s$ will do so, as in initial configurations $\alpha_{2}$ and $\alpha_{3}$ (the ones where the players face in the same direction) their initial distance of 2 is preserved and they never meet. The problem is therefore to find the mixed strategy $\mu$ which minimizes the expected meeting time. (The existence of such a strategy is guaranteed by the analysis given in [1].)

If the players $d o$ have a common sense of direction along the line, then we may take this direction to be player I's forward direction and, so, only average over the two cases $k=2,4$. The rendezvous value for this problem, $v^{\mathrm{c}}$, certainly cannot exceed $v$, as the players could choose to ignore this information. It has recently been observed by Shmuel Gal, and analyzed by Alpern and Gal [4], that if both players adopt $F B B$ as their first three moves and have not met by then, then they know that $k \in\{2,3\}$. That is, they know they both chose the same forward direction. Consequently, they are now playing the common-direction game $\Gamma^{\mathrm{c}}$, with value $v^{\mathrm{c}}$. It is widely thought that, in the symmetric rendezvous problem on the line, having a common sense of direction does not help the players. Weber [14] has shown that, on the triangle graph $K_{3}$, having a common notion of clockwise does not help the players, which result tends to lend credence to the following conjecture.

Conjecture 1. (The common-direction conjecture.) $v^{\mathrm{c}}=v$.

In the author's original paper [1] a very simple strategy was proposed (and naively conjectured to be optimal). In each three-period time interval, choose a random direction to call 'forward', and move $F B B$. If $k$ equals 2 or 3, the respective meeting times are 1 and 3 . In the 
remaining cases $(k=2,4)$, the players move in parallel and at the end of the last move they again face the original problem $\Gamma$. Hence, the expected meeting time for this mixed strategy is

$$
T=\frac{1}{4} \times 1+\frac{1}{4} \times 3+\frac{1}{2}(3+T), \quad \text { that is, } \quad T=5 .
$$

Subsequently, other authors have successively obtained lower expected meeting times by judicious choices of sets of longer move sequences (and appropriate probability distributions): Anderson and Essegaier [6] used $\{F F B B B, F B B B F F, F B B F B B, F B F B B B\}$ and Baston [8] used $\{F B B F F B B, F B B B F F F, F B F F F B B B F, F F B B B\}$. Recent work by Uthaisombut [13] and further work by Han et al. [11] has reduced the upper bound on $v$ to 4.574. The latter authors have conjectured that $v=v^{\mathrm{c}}=4.25$.

\section{Revealed information and partitions}

Thus far, the literature on rendezvous search theory has been restricted to the case in which the players each receive no additional information in the course of play - except, of course, that each has not yet met the other. Strategies are therefore simply paths, traveled under the assumption that no meeting has taken place. This is in contrast to most other dynamic games, in which information (about other player's actions or chance moves) is revealed at various stages. Cards being turned over constitute an important example of revealed information in parlor games.

Unlike Crawford and Haller [10], we consider games in which the players are given information feedback only once, at a preassigned time $n$. Take $n=2$, for example. Recalling our observation that players may begin with an $F$, there are only two move sequences of length 2 , namely $s_{1}=F F$ and $s_{2}=F B$. So the action set $\mathcal{A}=\mathcal{A}_{2}$, which describes all the player and Nature choices up to time 2 , is the $2 \times 2 \times 4=16$ element set $\{(i, j, k): i, j \in\{1,2\}, k \in$ $\{1,2,3,4\}\}$, where $(i, j, k)$ stands for the actions $s_{i}$ (for player I) and $s_{j}$ (for player II) and theinitial configuration $\alpha_{k}$. If the players have not met by time 2, they know that actual triple $(i, j, k)$ is one for which $T(i, j, k) \equiv T\left(s_{i}, s_{j}, \alpha_{k}\right)>2$. This subset of $\mathcal{A}_{2}$ is called the uncertainty set $u_{2}$.

The information we give the players after move 2 is described by a partition $\mathcal{P}$ of $U_{2}$. If $(i, j, k) \in U_{2}$ is played, then the players are told which element $P$ of $\mathcal{P}$ contains it. We call such a game $\Gamma(2, \mathcal{P})$ (or more generally $\Gamma(n, \mathcal{P})$, if they are told the element of the partition $\mathcal{P}$ of $\mathcal{U}_{n}$ after move $\left.n\right)$. The set $\mathcal{U}_{2}$ consists of the 10 elements with '?' as the entry in the following three-dimensional array $T(i, j, k)$, written with the third dimension, $k$, expressed as a $2 \times 2$ array in the ordering of (2):

\begin{tabular}{c|c|c|c|c|}
\multicolumn{1}{c}{} & \multicolumn{2}{c}{$s_{1}=F F$} & \multicolumn{2}{c}{$s_{2}=F B$} \\
\cline { 2 - 5 }$s_{1}=F F$ & $?$ & $?$ & $?$ & 2 \\
\cline { 2 - 5 } & $?$ & 1 & $?$ & 1 \\
\cline { 2 - 5 }$s_{2}=F B$ & $?$ & $?$ & $?$ & $?$ \\
\cline { 2 - 5 } & 2 & 1 & $?$ & 1 \\
\hline
\end{tabular}

That is, $U_{2}$ is the set

$\{(1,1,1),(1,1,2),(1,1,3),(1,2,1),(1,2,3),(2,1,1),(2,1,2),(2,2,1),(2,2,2),(2,2,3)\}$. 
When partitions of a fixed set are considered as a variable, the two to look at first are $\mathcal{P}^{2}$, the partition into one set, and $\mathcal{P}^{1}$, the partition into singletons. The first gives no new information, so $\Gamma\left(n, \mathcal{P}^{2}\right)$ is the same problem (game) as $\Gamma$. The second, $\mathcal{P}^{1}$, gives the players complete information, so the resulting subgame at time $n$ is easy to optimize: simply go towards the other player. Thus, if the distance between the players at time $n$ is $d=D\left(s_{i}, s_{j}, \alpha_{k}\right)$, they will optimally meet at time $n+d / 2$, so replacing all the '?' entries with $n+d / 2$ represents the best the players can do in each case $(i, j, k)$. In fact, this is what was done by Uthaisombut [13] to obtain a lower bound for $v$, the value of $\Gamma$, without regard to information structures. For $n=2$, this fills in the array (4) as follows:

\begin{tabular}{c|c|c|c|c|}
\multicolumn{1}{c}{$s_{1}=F F$} & \multicolumn{1}{c}{$s_{2}=F B$} \\
\cline { 2 - 5 }$s_{1}=F F$ & $2+\frac{6}{2}$ & $2+\frac{2}{2}$ & $2+\frac{4}{2}$ & 2 \\
\cline { 2 - 5 } & $2+\frac{2}{2}$ & 1 & $2+\frac{4}{2}$ & 1 \\
\cline { 2 - 5 }$s_{2}=F B$ & $2+\frac{4}{2}$ & $2+\frac{4}{2}$ & $2+\frac{2}{2}$ & $2+\frac{2}{2}$ \\
\cline { 2 - 5 } & 2 & 1 & $2+\frac{2}{2}$ & 1 \\
\hline
\end{tabular}

Averaging the values over $k=1,2,3,4$ gives a corresponding lower bound matrix for $\Gamma\left(2, \mathcal{P}^{1}\right)$ :

$$
B \equiv B\left(2, \mathcal{P}^{1}\right)=\frac{1}{4}\left(\begin{array}{ll}
12 & 11 \\
11 & 10
\end{array}\right)
$$

Now the players know they are playing $\Gamma\left(2, \mathscr{P}^{1}\right)$ and, so, facing the payoffs $B$. Hence, for $i=1,2$, they choose to play $s_{i}$ with probabilities $p_{i}$ such that the probability vector $p=\left(p_{1}, p_{2}\right)$ minimizes $p B p^{\top}$. Thus, the value of the game $\Gamma\left(2, \mathcal{P}^{1}\right)$ is given by

$$
V\left(2, \mathcal{P}^{1}\right)=\min _{p} p B p^{\top}=(0,1) B(0,1)^{\top}=2.5
$$

with the pure strategy $s_{2}=F B$ played exclusively. Since the players have (much) more information in the game $\Gamma\left(2, \mathcal{P}^{1}\right)$ than they did in the original game, $\Gamma$, we have the lower bound of [13],

$$
V(\Gamma) \geq 2.5
$$

Of course, since $\mathcal{P}^{1}$ is the finest partition, this will be the smallest lower bound obtainable using our methods. By analyzing coarser partitions, we will obtain higher lower bounds (without increasing $n$ ). However, to do this we must have methods of analyzing the subgames corresponding to the elements of the partitions that we consider. Some of the subgames that occur will be analyzed in the following section.

A third general partition that we refer to is the one that tells each player the actions of the other. We will call this partition $\mathcal{P}^{3}$. Its elements are the sets $U_{i, j}=\left\{\left(i^{\prime}, j^{\prime}, k\right) \in \mathcal{U}:\left(i^{\prime}, j^{\prime}\right)=(i, j)\right\}$, which reveal all player actions but no chance moves.

To summarize, we have defined three partitions of $\mathcal{U}=\mathcal{U}_{n}$, namely

$\mathcal{P}^{1}$ : partition into singletons, where all actions by chance and by players are revealed;

$\mathcal{P}^{2}$ : trivial partition into single element $U$, nothing is revealed; and

$\mathcal{P}^{3}$ : partition into sets $U_{i, j}$, where only player actions are revealed. 


\section{Analysis of the subgames $\Gamma^{*}, \bar{\Gamma}(d, e)$, and $\Gamma^{\mathrm{c}}$}

In the array (5), we have filled in certain entries as $n+d / 2$, where $d$ is the distance between the players at time $n$. The number $d / 2$ is trivially the value of the simple rendezvous problem where two players are placed a distance $d$ apart and told the direction to the other player. This game is too simple to be given a name, but for some other subgames that arise we give a name and analyze the best strategy and the corresponding rendezvous value.

The first subgame to be considered here, called $\Gamma^{*}$, is the symmetric game that arises from $\Gamma$ at time 1 if the players have not met by then. This is in fact a sufficient description of $\Gamma^{*}$, but for completeness we give an explicit definition as well.

Definition 1. In $\Gamma^{*}$ player I is placed at 0 , facing to the right. Player II is placed equiprobably in the following three positions: (i) at 2 , facing to the right; (ii) at -2 , facing to the right; and at -4 , facing to the left. The initial placement is identical from both players' points of view, so the setup is symmetric, and we require a symmetric solution.

We next consider a family of asymmetric subgames, $\bar{\Gamma}(d, e), d>e \geq 0$, which arise from the partition element $\{(i, j, 1),(i, j, 3)\}$; have $d=2+\triangle_{i}$ and $e=\left|\triangle_{j}\right|$, with

$$
\triangle_{i}=\hat{s}_{i}(n)
$$

the excess of $F$ s to $B \mathrm{~s}$ in the $n$-move sequence $s_{i}$; and take player II's starting position as the origin.

Definition 2. The game $\bar{\Gamma}(d, e), d>e \geq 0$, is played on a line labeled in the same way for each player, so each knows where they are. Player I is placed equiprobably at $\pm d$ and player II is placed equiprobably at $\pm e$.

In the next section we will need to know the rendezvous values of these two games. They are easy to calculate, as follows.

Lemma 1. Let $v$ denote the value of the original game, $\Gamma, v^{*}$ the value of $\Gamma^{*}, \bar{v}_{d, e}$ the value of $\bar{\Gamma}(d, e)$, and $v^{\mathrm{c}}$ the value of the common-direction game $\Gamma^{\mathrm{c}}$. Then

1. $v^{*}=\frac{4 v-4}{3}$,

2. $\bar{v}_{d, e}=\frac{3 d-e}{4}$ for $d>e$, and

3. $v^{\mathrm{c}} \geq 2 v-5$.

Proof. 1. We know by compactness [1] that $v$, the value of the original game, exists. We have shown that we can assume that the first move of both players is $F$. Consider the situation after one move. For initial configuration $\alpha_{4}(k=4)$, in which the players face each other, they meet in time $T=1$. For the remaining initial configurations $(k=1,2,3), T>1$. Consider the situation after one move from the point of view of, say, player II, who now views his current location as 0 and the direction he has just moved in as 'right'. If the initial configuration was $\alpha_{1}$, then from his point of view the location of player I is -4 and his forward direction must be left; if the initial configuration was $\alpha_{2}$, then player I must be at -2 with forward direction right; and if the initial configuration was $\alpha_{3}$, then player I must be at location +2 with forward direction right. Since the three possibilities for $k$ are equiprobable, player I's state relative to player II is as in $\Gamma^{*}$. The same is true for player II's state relative to player I. Thus, with probability $\frac{1}{4}$ 


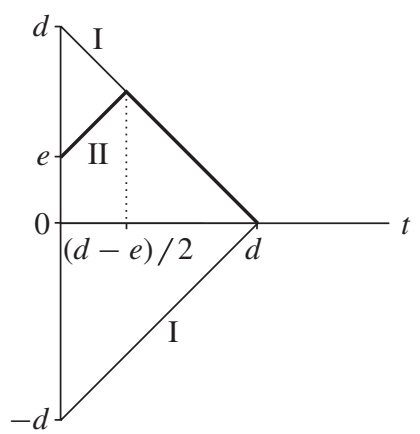

Figure 1: Optimal play for $\Gamma(d, e)$.

(for $k=4$ ) we have $T=1$ and with probability $\frac{3}{4}$ (for $k=1,2,3$ ) the minimum value of $T$ is $1+v^{*}$ (assuming optimal play in $\Gamma^{*}$ starting at real time 1 ). Hence, we have

$$
v=\frac{1}{4} \times 1+\frac{3}{4}\left(1+v^{*}\right), \quad \text { that is, } \quad v^{*}=\frac{4 v-4}{3} .
$$

2. Since $d>e$, player I knows (or can infer) the direction of player II (namely towards 0 ) and, hence, moves in that direction (indicated by the thin lines in Figure 1). The asymmetric solution is fairly obvious. Assuming (without loss of generality) that player II is placed at $+e$ on a vertical line, he goes up to meet one path of player I (starting at $+d$ ) and then follows that path down until it meets the other path of player I (starting at $-d$ ). This path is indicated by the thick line in Figure 1. In general, against a partner with a finite set of known paths (here the two possible paths of player I), the player's optimal strategy is such that he meets one of these paths as soon as possible, then another, and so on [2] (the same result appears as Theorem 16.10 of [3]). In the present situation, this means that player II either meets the path starting at $+d$ and then the one starting at $-d$ (the best strategy, as indicated by the thick line), or vice versa (which is worse). Consequently, with best play the players meet equiprobably at time $(d-e) / 2$ and at time $d$; hence,

$$
\bar{v}_{d, e}=\frac{1}{2}\left(\frac{d-e}{2}\right)+\frac{1}{2} d=\frac{3 d-e}{4} .
$$

3. Recall that $v^{\mathrm{c}}$ is the value of the common-direction game $\Gamma^{\mathrm{c}}$. Consider the strategy $F B B$ followed by optimal play in the common-direction game that results if no meeting takes place by time 3 . Clearly $v$ cannot exceed the expected meeting time for this strategy, so

$$
v \leq \frac{1}{4} \times 1+\frac{1}{4} \times 3+\frac{1}{2}\left(3+v^{\mathrm{c}}\right) .
$$

Solving for $v^{\mathrm{c}}$ gives the required inequality.

\section{Analysis of $\Gamma(n, \tilde{\mathcal{P}})$}

In this section we define an information partition $\tilde{\mathcal{P}} \equiv \tilde{\mathcal{P}}_{n}$ and find lower bounds for the subgames corresponding to its elements. The partition $\tilde{\mathcal{P}}$ refines the partition $\mathcal{P}^{3}$ (see (6)), namely that which only reveals player actions. For $n=2, \mathscr{P}^{3}$ is the partition of the 10 elements of $\mathcal{U}_{2}$ (the '?'s) into the four $2 \times 2$ arrays in (4). Thus, in the partition $\tilde{\mathcal{P}}$ the players are told the moves of the other player and perhaps something about the chance moves. To define $\tilde{\mathcal{P}}$ we therefore have to define a further partition of each element $U_{i, j}$ of $\mathcal{P}^{3}$. 
To this end, fix $n$ and for convenience number the $2^{n-1} n$-move strategies $s_{i}, i=1, \ldots$, $2^{n-1}$ (since they all start with $F$ ), so that if $i<j$ then a player using $s_{i}$ and facing their partner, who is facing in the same direction (initial configuration $\alpha_{2}$ ), will catch their partner by time $n$. That is,

$$
T(i, j, 2) \leq n, \quad \text { or, equivalently, } \quad(i, j, 2) \notin \mathcal{U}, \quad \text { for } i<j .
$$

For example, for $n=3$ we have

$$
s_{1}=F F F, \quad s_{2}=F F B, \quad s_{3}=F B F, \quad s_{4}=F B B .
$$

By symmetry, we need only analyze those $i$ and $j$ with $i \leq j$. A particular ordering of the $s_{i}$ which satisfies (9) and in fact gives (10) for $n=3$ can be defined as follows: let $\gamma \equiv \gamma\left(s, s^{\prime}\right):=\min \left\{r: s(r) \neq s^{\prime}(r)\right\}$ (where $\hat{s}$ is as defined in (3)) and use the order ' $\prec$ ', such that $s \prec s^{\prime}$ if and only if $s(\gamma)=F$. We are listing the $s_{i}$ in dictionary (lexicographic) order (although with the convention that $F$ appears lexicographically before $B$ ).

Note that, since $T(i, j, 4)=1$ for all $i$ and $j$, the set $U_{i, j}$ is at most the three-element set $\exists$, using the two-dimensional ordering of (2). Furthermore, for $i<j, U_{i, j}$ is at most $\Theta$ (the left-hand column), according to (9).

We are now in a position to describe how $\tilde{\mathcal{P}}$ partitions each set $U_{i, j}$. This depends on $i$ and $j$ in four combinations described in the following cases.

Case 1: $U_{i, j}=\{(i, j, k)\}$, a singleton, for some $k$. In this case each player knows the state of the game, namely $(i, j, k)$, and knows the direction and the distance,

$$
\triangle_{i, j, k}=D\left(s_{i}, s_{j}, \alpha_{k}\right)>0,
$$

to the other player. Consequently, they will move at unit speed towards each other and their meeting time will be $n+\triangle_{i, j, k} / 2$. Hence, if player I chooses path $s_{i}$ and player II chooses path $s_{j}$, then $b_{i, j}$, their expected meeting time in the game $\Gamma(n, \tilde{\mathcal{P}})$, is given by

$$
4 b_{i, j}=n+\frac{\triangle_{i, j, k}}{2}+\sum_{k^{\prime} \neq k} t_{i, j, k^{\prime}}, \quad \text { where } t_{i, j, k}=T\left(s_{i}, s_{j}, \alpha_{k}\right) .
$$

In the remaining cases we assume that $U_{i, j}$ has at least two elements, and we describe how they are partitioned in $\tilde{\mathcal{P}}$.

Case 2: $i<j$. By (9) and the fact that $t_{i, j, 4}=1$ for all $i, j$, we have

$$
U_{i, j}=\{(i, j, 1),(i, j, 3)\},
$$

which we draw as follows to illustrate the fact that if we take $U_{i, j}$ to be a single element of $\tilde{\mathcal{P}}$ then player I (corresponding to the arrows on the left) knows that player II is behind him (in his backwards direction):

$$
\begin{array}{|l|}
\leftarrow \leftarrow \rightarrow \\
\leftarrow \leftarrow
\end{array} \quad(k=1,3)
$$

Both know that player I's location at time $n$ is a distance $d=2+\triangle_{i}$ from player II's starting location, and that player II is a distance $e=\left|\triangle_{j}\right|$ from his own starting location. Hence, they know they are playing the game $\bar{\Gamma}\left(2+\triangle_{i}, \triangle_{j}\right)$, with value $\bar{v}_{2+\triangle_{i}, \triangle_{j}}$, and in this case the meeting times corresponding to the four initial configurations $k$ are

\begin{tabular}{|c|c|}
\hline$n+\bar{v}_{2+\triangle_{i}, \triangle_{j}}$ & $t_{i, j, 2}$ \\
\hline$n+\bar{v}_{2+\triangle_{i}, \triangle_{j}}$ & $1=t_{i, j, 4}$ \\
\hline
\end{tabular}


and the expected meeting time, $b_{i, j}$, satisfies

$$
4 b_{i, j}=2\left(n+\bar{v}_{2+\triangle_{i}, \triangle_{j}}\right)+t_{i, j, 2}+1 .
$$

Case 3: $i=j$ with $t_{i, i, 1} \leq n$. Note that if both players use the same move sequence $s_{i}$, they will not meet if they are facing the same direction $(k=2,3)$, so certainly $U_{i, i}=$ $\{(i, i, 2),(i, i, 3)\}$. In this case we take $U_{i, i}$ to be an element of $\tilde{\mathcal{P}}$. If the players are told that they are in $U_{i, i}$, they can conclude they are still a distance 2 apart and have moved in the same direction. Hence, they are playing the common-direction game $\Gamma^{\mathrm{c}}$ and with best play will meet in additional time $v^{\mathrm{c}}$. Hence, the entries of the $(i, i)$ arrays of this type for $\Gamma(n, \tilde{\mathcal{P}})$ are

\begin{tabular}{|c|c|}
\hline$t_{i, i, 1}$ & $n+v^{\mathrm{c}}$ \\
\hline$n+v^{\mathrm{c}}$ & 1 \\
\hline
\end{tabular}

and $4 b_{i, i}=t_{i, i, 1}+2 n+2 v^{\mathrm{c}}+1$.

Case 4: $i=j$ with $t_{i, i, 1}>n$. Here we consider two subcases. First let $\left|\triangle_{i}\right| \neq 1$. Since $t_{i, i, 1}>n$, we have $U_{i, i}=\{(i, i, 1),(i, i, 2),(i, i, 3)\}$. Since $t_{i, i, 1}>n$, we must also have $\triangle_{i} \geq 0$. There are two reasonable ways to partition the three-element set

$$
U_{i i}=\begin{array}{|l|l|}
\leftarrow \quad \rightarrow & \rightarrow \rightarrow \rightarrow \\
\hline \leftarrow \quad \leftarrow &
\end{array}
$$

namely (in a notation that will become clear below)

$$
\begin{array}{|l|l|l|l|l|}
\hline \text { (a) } & \text { (b) with } \\
\text { (a) } & k \in\{\{1,3\},\{2\}\} \quad \text { and } \quad \text { (c) } & \text { (d) with } k \in\{\{1\},\{2,3\}\} \text {. } \\
\hline
\end{array}
$$

Consider the first (left-hand) partition. If the players are told (a) that they are in the set $\{(i, i, 1),(i, i, 3)\}$, then they can conclude, as above, that they are playing the asymmetric game $\bar{\Gamma}\left(\triangle_{i}+2, \triangle_{i}\right)$, with value $\bar{v}_{\triangle_{i}+2, \triangle_{i}}=\left(1+\triangle_{i}\right) / 2$. If they are told (b) that they are in $\{(i, i, 2)\}$, then they are still a distance 2 apart and can meet in additional time 1 . Thus, the sum of the additional meeting times in the three cases $(k=1,2,3)$ is

$$
2 \frac{1+\triangle_{i}}{2}+1=2+\triangle_{i}
$$

Now consider the second (right-hand) partition. If the players are told (c) that they are in the set $\{(i, i, 1)\}$, then they each know the other's direction and that their distance apart at time $n$ is $\triangle_{i, i, 1}=2+2 \triangle_{i}$, meaning that they can meet in additional time $1+\triangle_{i}$. If they are told (d) that they are in $\{(i, i, 2),(i, i, 3)\}$, then they are in the common-direction game $\Gamma^{\mathrm{c}}$, so the additional time is $v^{\mathrm{c}}$. Hence, the sum of the three additional times is

$$
1+\triangle_{i}+2 v^{\mathrm{c}}
$$

Since we are trying to maximize the entries (meeting times), we take the first set as our partition of $U_{i, i}$ if and only if $2+\triangle_{i} \geq 1+\triangle_{i}+2 v^{\mathrm{c}}$ or, equivalently, $1 \geq 2 v^{\mathrm{c}}$, which is false. (Note that certainly $v^{\mathrm{c}} \geq \frac{5}{3}$, the revealed information value (see $(1)$ ), and that $v^{\mathrm{c}} \geq \frac{13}{4}$, the asymmetric-rendezvous value [2].) Therefore, in the partition $\tilde{\mathcal{P}}$ we use the second partition of $U_{i, i}$ in (11). It follows that $4 b_{i, i}$ is the sum of the four entries corresponding to the values of $k=1,2,3,4$ in

\begin{tabular}{|c|c|}
\hline$n+1+\triangle_{i}$ & $n+v^{\mathrm{c}}$ \\
\hline$n+v^{\mathrm{c}}$ & 1 \\
\hline
\end{tabular}


so in this subcase

$$
4 b_{i, i}=3 n+2 v^{\mathrm{c}}+2+\triangle_{i} .
$$

In the second subcase of case 4 we let $\left|\triangle_{i}\right|=1$. Note that this implies that $n$ is odd. As in the previous case, we have $U_{i, i}=\{(i, i, 1),(i, i, 2),(i, i, 3)\}$. Take $U_{i, i}$ to be an element of $\tilde{\mathcal{P}}$. If the players are told that they are in this element of $\tilde{\mathcal{P}}$, they can conclude that they are in the symmetric game $\Gamma^{*}$ defined in the previous section and, hence, that the minimal remaining expected time until they meet is $v^{*}$. In this subcase we thus have

$$
4 b_{i, i}=3\left(n+v^{*}\right)+1 .
$$

Observe that this is a larger value than that in the previous case (see (13)), as

$$
\begin{aligned}
(3(n+ & \left.\left.+v^{*}\right)+1\right)-\left(3 n+2 v+2+\triangle_{i}\right) \\
& \geq\left(3\left(n+\frac{4 v-4}{3}\right)+1\right)-(3 n+2 v+2) \\
& =2 v-5 \\
& >2 \times \frac{13}{4}-5 \\
& >0
\end{aligned}
$$

\begin{tabular}{|c|c|c|c|c|c|c|c|c|}
\hline \multirow{3}{*}{$s_{1}=F F F$} & \multicolumn{2}{|c|}{$s_{1}=F F F$} & \multicolumn{2}{|c|}{$s_{2}=F F B$} & \multicolumn{2}{|c|}{$s_{3}=F B F$} & \multicolumn{2}{|c|}{$s_{4}=F B B$} \\
\hline & $3+\frac{8}{2}$ & $3+v^{\mathrm{c}}$ & $3+\bar{v}_{5,1}$ & 3 & $3+\bar{v}_{5,1}$ & 2 & $3+\bar{v}_{5,1}$ & 2 \\
\hline & $3+v^{\mathrm{c}}$ & 1 & $3+\bar{v}_{5,1}$ & 1 & $3+\bar{v}_{5,1}$ & 1 & $3+\bar{v}_{5,1}$ & 1 \\
\hline \multirow{2}{*}{$s_{2}=F F B$} & & & $3+v^{*}$ & $3+v^{*}$ & $3+\bar{v}_{3,1}$ & 2 & $3+\bar{v}_{3,1}$ & 2 \\
\hline & & & $3+v^{*}$ & 1 & $3+\bar{v}_{3,1}$ & 1 & $3+\bar{v}_{3,1}$ & 1 \\
\hline \multirow{2}{*}{$s_{3}=F B F$} & & & & & $3+v^{*}$ & $3+v^{*}$ & $3+\bar{v}_{3,1}$ & 3 \\
\hline & & & & & $3+v^{*}$ & 1 & $3+\bar{v}_{3,1}$ & 1 \\
\hline \multirow{2}{*}{$s_{4}=F B B$} & & & & & & & 3 & $3+v^{\mathrm{c}}$ \\
\hline & & & & & & & $3+v^{\mathrm{c}}$ & 1 \\
\hline
\end{tabular}

since $v$, the value of the symmetric game $\Gamma$, cannot be smaller than the value, $\frac{13}{4}$, of the corresponding asymmetric game (see [2] or Theorem 9 of [3]).

For the ordering (10), which holds for $n=3$, the partition and the numbers making up $b_{i, j}$ can be seen from the following array, where, by symmetry of the players (and the matrix $B$ ), we can confine our analysis to strategy pairs $i \leq j$ :

The set $\mathcal{U}_{3}$ consists of all the entries which are not written as a single number (including the entry $3+\frac{8}{2}$ ). The partition, $\tilde{\mathcal{P}}$, of the nonnumeric entries in each $2 \times 2$ array is based on identical-form entries.

If we combine all the cases, use (7) and (8) to evaluate $v^{*}$ and $\bar{v}$ in terms of $v$, and assume the common-direction conjecture $\left(v^{\mathrm{c}}=v\right)$ to hold, we obtain the following theorem.

Theorem 1. Let $\tilde{\mathcal{P}}$ be the partition of $\mathcal{U}_{n}$ described above, and let $\Gamma(n, \tilde{\mathcal{P}})$ be the game in which the players play symmetrically as in $\Gamma$ for $n$ moves and, if they have not met by that time, 
are then told the element of the $\tilde{\mathcal{P}}$ to which the action triple $(i, j, k)$ belongs. If, based on this information, the players choose n-move strategies $s_{i}$ and $s_{j}$ and subsequently play optimally, then, assuming the common-direction conjecture to hold, for $i<j$ the expected time to meet, $\tilde{b}_{i, j}$, is given by

$$
4 \tilde{b}_{i, j}= \begin{cases}2 n+\frac{3 \triangle_{i}-\left|\triangle_{j}\right|}{2}+t_{i, j, 2}+4, & i<j, t_{i, j, 1}>n, t_{i, j, 3}>n, \\ n+\frac{\triangle_{i}+\triangle_{j}+2}{2}+t_{i, j, 2}+t_{i, j, 3}+1, & i<j, t_{i, j, 1}>n, t_{i, j, 3} \leq n, \\ n+\frac{\triangle_{i}-\triangle_{j}}{2}+t_{i, j, 1}+t_{i, j, 2}+2, & i<j, t_{i, j, 1} \leq n, t_{i, j, 3}>n, \\ t_{i, j, 1}+t_{i, j, 2}+t_{i, j, 3}+1, & i<j, t_{i, j, 1} \leq n, t_{i, j, 3} \leq n, \\ t_{i, i, 1}+2(n+v)+1, & i=j, t_{i, i, 1} \leq n, \\ 3 n+2 v+2+\triangle_{i}, & i=j, t_{i, i, 1}>n,\left|\triangle_{i}\right| \neq 1, \\ 3 n+4 v-3, & i=j, t_{i, i, 1}>n,\left|\triangle_{i}\right|=1 .\end{cases}
$$

The matrix $\tilde{B} \equiv \tilde{B}_{n}=\left\{\tilde{b}_{i, j}\right\}$ is symmetric. It has entries which are constants or linear functions of $v$ with positive coefficients. The (rendezvous) value, $\tilde{v} \equiv \tilde{v}_{n}$, of the symmetric rendezvous problem $\Gamma(n, \tilde{\mathcal{P}})$ is given by

$$
\tilde{v}_{n}=\min _{\left\{p: p_{i} \geq 0, \sum p_{i}=1\right\}} p \tilde{B}_{n} p^{\top} \leq v
$$

If we do not assume the common-direction conjecture to hold, then the result remains the same, except with $v$ replaced by $v^{\mathrm{c}}$ in the next-to-last case in the definition of $\tilde{b}_{i, j}$.

We illustrate the construction of $\tilde{B}_{n}$ and the calculation of $\tilde{v}_{n}$ by considering the case $n=3$ with the strategy ordering $s_{1}=F F F, s_{2}=F F B, s_{3}=F B F, s_{4}=F B B$. Using the formula for $\tilde{b}_{i, j}$ given above, and array (14), we obtain

$$
\begin{aligned}
\tilde{B} \equiv \tilde{B}(v) & =\frac{1}{4}\left(\begin{array}{cccc}
2 v^{\mathrm{c}}+14 & 17 & 16 & 16 \\
17 & 4 v+6 & 13 & 13 \\
16 & 13 & 4 v+6 & 14 \\
16 & 13 & 14 & 2 v^{\mathrm{c}}+10
\end{array}\right) \\
& =\frac{1}{4}\left(\begin{array}{cccc}
2 v+14 & 17 & 16 & 16 \\
17 & 4 v+6 & 13 & 13 \\
16 & 13 & 4 v+6 & 14 \\
16 & 13 & 14 & 2 v+10
\end{array}\right),
\end{aligned}
$$

under the common-direction conjecture.

We want to find $\tilde{v} \equiv \tilde{v}_{3}$, that is,

$$
\tilde{v}_{n}(v) \equiv g(v)=\min _{p} p \tilde{B} p^{\top}, \quad n=3,
$$

where the minimum is taken over probability 4-vectors $p$, in terms of $v$. In particular, under the common-direction hypothesis $\left(v^{\mathrm{c}}=v\right)$ we find the unique minimizing $p$ to have the components

$$
p_{1}=0, \quad p_{2}=\frac{4 v-5}{16 v-26}, \quad p_{3}=\frac{4 v-7}{16 v-26}, \quad p_{4}=\frac{8 v-14}{16 v-26},
$$


yielding

$$
g(v)=\frac{16 v^{2}+160 v-303}{4(16 v-26)} .
$$

Since the game $\Gamma(3, \tilde{\mathcal{P}})$ gives the players more information than does $\Gamma$, we have $v \geq g(v)$, which holds for

$$
v \geq \frac{11+2 \sqrt{5}}{4} \approx 3.868 .
$$

So, assuming the common-direction conjecture to hold, we have $v \geq 3.868$. The corresponding estimate if we reveal everything (that is, use partition $\mathcal{P}^{1}$ ) at time $n=3$ is $v \geq 3$, as obtained by Uthaisombut [13]. A related bound of $v^{\mathrm{c}}>3.5869$ for $n=3$ has been obtained by Han et al. [11] (under the assumption that the players have a common notion of direction along the line) by revealing everything if different move sequences are used and telling the players that they have used the same move sequence, given that that has occurred. Substituting this lower bound of [11] for $v^{\mathrm{c}}$ for $n=3$ into (15) gives the bound

$$
\tilde{v}_{3} \geq 3.829,
$$

which is obtained by considering only move sequences of length 3 (whether or not there is a common notion of direction). By formulating a relaxed version of the problem as one of semidefinite programming, Han et al. [11] were able to handle the large matrices that occur for $n \leq 7$, obtaining for $n=7$ the bound $v^{\mathrm{c}} \geq 4.1520$. Bootstrapping their estimates of $v^{\mathrm{c}}$ obtained for these $n$ would give higher lower bounds for $\tilde{v}_{n}$ in each case, but apparently the improvement lessens for greater values of $n$. In a literal sense, we could use these bounds to obtain better estimates for $\tilde{v}_{3}$, but this would be a rather unfair estimate for comparison, as they require the analysis of $n$-move sequences (that is, long ones), while (16), as mentioned above, requires only the analysis of 3-move sequences.

\section{Partitions with incomplete information subgames}

We now consider revealed information variants of the symmetric rendezvous problem on the line, without the assumption that $\mathcal{P}$ refines $\mathcal{P}^{3}$. That is, we do not assume that each player is given information which includes the past actions of his partner. If the game is stopped at time $n$, then without any revealed information the private knowledge of player I is simply the $n$-move strategy sequence he has used, namely $i$ (corresponding to the path $s_{i}$ ). He does not know the other player's strategy sequence, $j$, nor Nature's move, $k$. If the partition $\mathcal{P}$ does refine $\mathcal{P}^{3}$, then the subgames $\Gamma(n, P), P \in \mathcal{P}$, all have complete information.

Suppose, however, that $\mathcal{P}$ has an element (set) $P$ which contains elements of the information space $U_{n}$ with distinct player-I strategies, say $i$ and $i^{\prime}$. In the subgame $\Gamma(n, P)$ corresponding to $P$, player I knows his type ( $i$ or $i^{\prime}$ ), since he knows his previous moves, but player II does not know this. The subgame therefore has incomplete information. However, given the common distribution $p$ over $n$-move strategies that both players are employing, each can update the distribution of the other's type. The game $\Gamma(n, P)$ is thus a Bayesian game.

For example, when $n=3$ the elements of $U_{3}$ for $i=1(F F F), i=2(F F B)$, and $j=4$ $(F B B)$ are the four elements $(1,4,1),(1,4,3),(2,4,1)$, and $(2,4,3)$. In the partition $\tilde{\mathcal{P}}$ of the previous section, these four elements were partitioned into two sets, $\{(1,4,1),(1,4,3)\}$ and $\{(2,4,1),(2,4,3)\}$. That is, $i$ and $j$ were revealed, but not $k$. Recall that in the first instance this led to the subgame $\Gamma(5,1)$, with value $\bar{v}_{5,1}=\frac{7}{2}$, and in the second instance to $\Gamma(3,1)$, with value $\bar{v}_{3,1}=2$. In both games player I knows the direction to player II, so to him the distance 


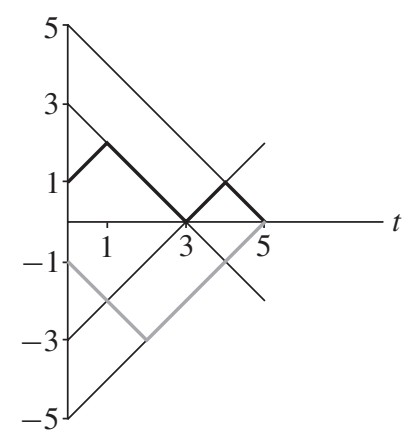

FIGURE 2: Player II knows player I starts at \pm 3 or \pm 5 .

is strategically irrelevant: he simply moves in that direction. How does this uncertainty affect player II? Let $p_{1}$ and $p_{2}$ denote the respective probabilities with which player I chooses paths $s_{1}$ and $s_{2}$. Player II knows that with probability $p_{1}$ player I is starting the subgame a distance 5 (in either direction) from player II's starting point in the original game. Moreover, player II is a distance 1 from that point (and knows what direction it is in).

Therefore, player I's four possible paths start at \pm 5 or \pm 3 , as indicated by the four thin lines in Figure 2. If $p_{1}$ is sufficiently large then player II clearly should take the line (which we have drawn starting at -1 ) which minimizes the expected time to reach the equiprobable paths of player I starting from \pm 5 . Similarly, if $p_{2}$ is large he should take the thick line which we have drawn starting at +1 . (We could have drawn both paths starting from the same point, but it would have been less clear.) The expected meeting times for the two paths are given by

$$
\begin{array}{ll}
p_{1}\left(\frac{1}{2} \times 4+\frac{1}{2} \times 5\right)+p_{2}\left(\frac{1}{2} \times 1+\frac{1}{2} \times 3\right)=\frac{9 p_{1}+4 p_{2}}{2} \text { for the black line, } \\
p_{1}\left(\frac{1}{2} \times 2+\frac{1}{2} \times 5\right)+p_{2}\left(\frac{1}{2} \times 1+\frac{1}{2} \times 4\right)=\frac{7 p_{1}+5 p_{2}}{2} \quad \text { for the gray line. }
\end{array}
$$

Consequently, player II should use the 'black' strategy if $p_{2} \geq 2 p_{1}$ and the 'gray' strategy otherwise.

Now consider the partition $\tilde{\mathcal{P}}$ indicated by identical forms in (14), and change it so that the twelve cells with entry either $3+\bar{v}_{5,1}$ or $3+\bar{v}_{3,1}$ together form a single element of a new partition. Player II can calculate the probability, $q_{1}$, that $d=5$ and the probability, $q_{2}$, that $d=3$ (in the subgame $\bar{\Gamma}(d, e)$ ) based on the strategy sequence, $j$, that he has used up to time 3. For instance, if he has used strategy sequence $j=4$, then $q_{1}=p_{1} /\left(p_{1}+p_{2}+p_{3}\right)$. This partition (and generalizations to higher $n$ ) gives player II less information than he had in $\Gamma\left(n, \mathcal{P}^{3}\right)$, and cannot result in smaller rendezvous values. For $n=3$ it is of no help, because $p_{1}=0$ in the optimum probability 4 -vector, but in general this idea will give higher lower bounds than can be obtained using the $b_{i, j}$ from Theorem 1 .

\section{Conclusions}

Giving the rendezvousers some (revealed) information during the course of their search can be used both to describe real problems, where such information is available or can be sent, and to obtain lower bounds on rendezvous values of existing problems which have no such information. In the latter case the information partition $\mathcal{P}$, describing the information to be given at time $n$, must be carefully chosen to be as coarse as possible while still having all its 
subgames $\Gamma(n, P), P \in \mathcal{P}$, amenable to analysis. This will probably be a useful technique for certain rendezvous problems and not for others.

As observed by an anonymous referee, the information revelation described in this paper is not the only sort that might be considered. We could also consider a situation in which the revealed information depends on the mixed strategy chosen by the rendezvous team, although such a model would take us out of the usual game tree description.

\section{References}

[1] Alpern, S. (1995). The rendezvous search problem. SIAM J. Control Optimization 33, 673-683.

[2] Alpern, S. And Gal, S. (1995). Rendezvous search on the line with distinguishable players. SIAM J. Control Optimization 33, 1270-1276.

[3] Alpern, S. and Gal, S. (2003). The Theory of Search Games and Rendezvous (Internat. Ser. Operat. Res. Manag. Sci. 55). Kluwer, Boston, MA.

[4] Alpern, S. and Gal, S. (2006). Two conjectures on rendezvous in $K_{3}$. Res. Rep. LSE-CDAM-2006-21, Department of Mathematics, London School of Economics. Available at http://www.cdam.lse.ac.uk/Reports/ reports2006.html.

[5] Alpern, S., Baston, V. and Essegaier, S. (1999). Rendezvous search on a graph. J. Appl. Prob. 36, $223-231$.

[6] Anderson, E. J. AND Essegaier, S. (1995). Rendezvous search on the line with indistinguishable players. SIAM J. Control Optimization 33, 1637-1642.

[7] Anderson, E. J. And Weber, R. R. (1990). The rendezvous problem on discrete locations. J. Appl. Prob. 28, 839-851.

[8] Baston, V. J. (1999). Two rendezvous search problems on the line. Naval Res. Logistics 46, 335-340.

[9] Baston, V. And GaL, S. (2001). Rendezvous search when marks are left at the starting points. Naval Res. Logistics 48, 722-731.

[10] Crawford, V. P. ANd Haller, H. (1990). Learning how to cooperate: optimal play in repeated coordination games. Econometrica 58, 571-596.

[11] Han, Q., Du, D., Vera, J. and Zuluaga, L. F. (2006). Improved bounds for the symmetric rendezvous value on the line. To appear in Operat. Res.

[12] Lim, W. S., Alpern, S. And Beck, A. (1997). Rendezvous search on the line with more than two players. Operat. Res. 45, 357-364.

[13] Uthaisombut, P. (2006). Symmetric rendezvous search on the line using move patterns with different lengths. Submitted.

[14] Weber, R. R. (2006). The optimal strategy for symmetric rendezvous search on $K_{3}$. Preprint, Statistical Laboratory, University of Cambridge. Available at http://www.statslab.cam.ac.uk/ rrw1/research/weber-k3.pdf. 